\title{
The RAPSODI project: SiPM development for applied research in radiation protection
}

\author{
Chiara Cappellini, Massimo Caccia, Valery Chmill, Antonio Bulgheroni, Christian Pychlau, Jan U. Wuerfel,
} Alexander Martemiyanov, Alexander Golubev, Aleksei Kantsyrev, Nikolai Markov and Natalia Fedyushkina

\begin{abstract}
RAPSODI is a research project funded by the European Commission within the sixth framework program. The aim of the project is the development of a set of radiation detectors for three well defined applications based on Silicon Photo-Multipliers (SiPM), representing the state-of-the-art in single photon sensitive detectors. This paper focuses on the currently most advanced RAPSODI application, namely in-vivo real-time dosimetry in mammography.
\end{abstract}

Index Terms-SiliconPhotoMultipliers, Mammography

\section{INTRODUCTION}

M AMMOGRAPHY identifies the radiographic examination of the human breast, targeted to the early diagnostic of different types of tumours, cysts and microcalcifications. Mammography is the only imaging technique that has been proven to reduce mortality from breast cancer. Hence, together with self-breast and physician examination it is an essential part of regular breast care. In some countries, routine mammography of women is encouraged every 1 to 5 years . It is clear that knowing the actual dose released to the patient during the irradiation is of extreme importance, especially in case of screening procedures. Within the RAPSODI project, funded by the EC in the Sixth Framework Program $^{1}$, a novel real time dosimeter for mammography has been developed. The results of the feasibility study are reported in the following.

\section{CONCEPTUAL DESIGN AND PROTOTYPE CHARACTERISTICS}

The device being developed is a novelty in the field of mammography. It is a cost-effective and compact instrument, consisting in sensitive volume made out of scintillating material, a Silicon Photomultiplier light sensor and a small local display of the recorded dose. The sensitive volume is being engineered not to introduce any artifacts in the image and the instrument, installed on the paddle of the mammography X-ray machine, complies the safety regulations with no electrical elements in the proximity of the patient. A

C. Cappellini, M. Caccia and V. Chmill are with Dipartimento di Fisica e Matematica, Università dell'Insubria, Via Valleggio 11, 22100 Como, Italy.

A. Bulgheroni is with I.N.F.N. - Sez. di Milano, via Celoria 16, 20133 Milano, Italy.

C. Pychlau and J. U. Wuerfel are with PTW-Freiburg PhysikalischTechnische Werkstaetten Dr. Pychlau GmbH, Loerracher Str. 7, D-79115 Freiburg, Germany.

A. Martemiyanov, A. Golubev, A. Kantsyrev, N. Markov and N. Fedyushkina are with SSC RF ITEP, Bolshaya Cheremushkinskaya, 25117218 Moscow Russia.

${ }^{1}$ Contract Number: COOP 32993 - RAPSODI patent application for the intellectual property protection of the inventive idea has been deposited ${ }^{2}$.

Two prototypes were characterized:

- a blue light emitting plastic tile, optically coupled to a wavelength shifting (WLS) fiber, in order to match the SiPM peak sensitivity.

- a green emitting scintillating fiber, coupled to a clera fiber conveying the light to a SiPM.

For the tile, the SiPM sensor in use was produced by $\mathrm{SensL}^{3}$, a RAPSODI consortium partner. The fiber system was interfaced to both the SensL sensor and to a Multi Pixel Photon Counter produced by HAMAMATSU ${ }^{4}$. The basic characteristics of the sensors are reported in Tab I.

The charge from the sensor is integrated and sampled by a QDC after a transimpedance amplification stage. The QDC conversion capacitance is $0.11 \mathrm{pC}$ per QDC unit.

\begin{tabular}{lcc}
\hline \hline & SensL & Hamamatsu \\
\hline Number of Diodes: & 8640 & 400 \\
Area: & $3 \mathrm{~mm} \times 3 \mathrm{~mm}$ & $1 \mathrm{~mm} \times 1 \mathrm{~mm}$ \\
Diode dimension: & $20 \mu \mathrm{m} \times 20 \mu \mathrm{m}$ & $50 \mu \mathrm{m} \times 50 \mu \mathrm{m}$ \\
Breakdown Voltage: & $28.04 \mathrm{~V}$ & $70.56 \mathrm{~V}$ \\
Dark Count Rate: & $8 \mathrm{MHz}$ & $270 \mathrm{kHz}$ \\
Optical Crosstalk: & $22.5 \%$ & $10 \%$ \\
Gain: & $10^{6}$ & $7.5 \cdot 10^{5}$ \\
PDE: & $4 \%(\lambda=520 \mathrm{~nm})$ & $50 \%(\lambda=400 \mathrm{~nm})$ \\
\hline \hline
\end{tabular}

TABLE I

SIPM MAIN CHARACTERISTICS. MEASUREMENTS REFER TO ROOM TEMPERATURE AND A WORKING POINT OF + 2 V WITH RESPECT TO THE BREAKDOWN VOLTAGE.

\section{EXPERIMENTAL RESULTS}

The prototype has been characterized at the RAPSODI partner company PTW-Freiburg ${ }^{5}$, exposing the prototype to the X-ray beam of a reference mammography unit. Dose rates spanning from less than $1 \mathrm{mGy} / \mathrm{s}$ to a few hundreds $\mathrm{mGy} / \mathrm{s}$ have been used to irradiate the device in realistic conditions. For each irradiation, the effective dose rate has simultaneously been measured with a calibrated sensor ${ }^{6}$ together with the

\footnotetext{
${ }^{2}$ EPO Patent nr. 08015371.1-1240
}

${ }^{3}$ SensL, Lee House, Riverview Business Park Bessboro Road Blackrock, Cork

${ }^{4}$ http://www.hamamatsu.com/

${ }^{5}$ PTW-Freiburg, Lörracher Strasse 7, 79115 Freiburg, Germany. http://www.ptw.de/

${ }^{6}$ UNIDOS, a ultra high sensitive electrometer for charge and current measurement, produced by PTW-Freiburg 


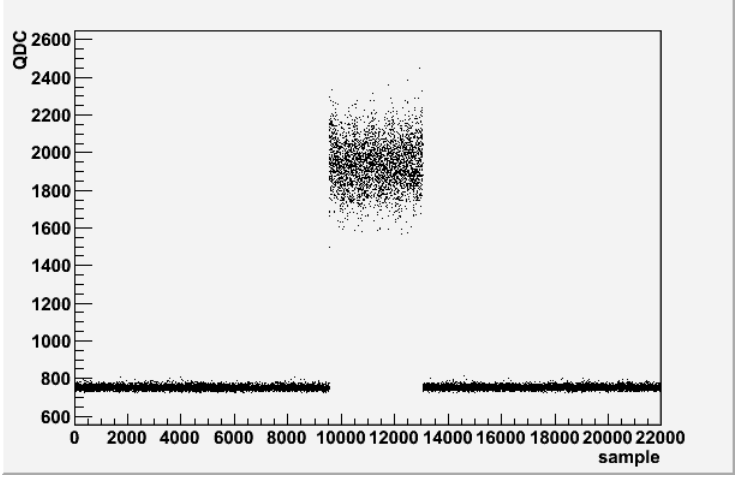

Fig. 1. The output signal for a typical data set is shown as a function of the sample number, rescaled in time units. The integration time for the reported data was $3000 \mathrm{~ns}$ for a dose rate of $21 \mathrm{mGy} / \mathrm{s}$.

irradiation time ${ }^{7}$. The signal has been typically sampled at a frequency of $30 \mathrm{kHz}$ with integration times ranging between $500 \mathrm{~ns}$ and $5000 \mathrm{~ns}$ and exposure times of $\approx 0.1 \mathrm{~s}$ and $\mathrm{a}$ simultaneus measurement of both the dose rate and the total delivered dose. The typical output signal from the prototype is displayed in Fig. 1, where the samples corresponding to the tile irradiation are clearly distinguishable.

The characterization of the prototype has been performed relying on four main figures of merit:

- precision, defined as the standard deviation of the measurements for constant irradiation conditions.

- sensitivity, namely the minimum detectable variation in the dose rate.

- minimum detectable signal, differing from the sensitivity since it accounts as well for the spread in the offset

- linearity, measured against the dose rate in the region of interest.

Figures have been evaluated on the same data set referring to the following measured quantities (see also Fig. 1 and Fig. 2):

- the overall sum, defined as the sum of all of the recorded signals, offset subtracted, including the beam-off samples as well. In the absence of systematic errors in the offset calculation, beam-off events average to zero without any bias on the sum of the beam-on samples. Actually, the overall sum is the minimum bias estimate of the signal, even if the precision is possibly lower because of the sum on the maximum number of samples.

- the edge sum, evaluated as the sum of all of the events occurring between two well defined edges. Edges are identified requiring $\mathrm{N}$ consecutive offset subtracted samples to exceed $M$ times the offset spread. A typical value of $\mathrm{M}$ and $\mathrm{N}$ is 3 . The edge sum is expected to provide a high precision, with a possible bias on the total integrated signal depending on the edge definition.

- the buffered sum, computed as the sum of all of the events occurring between the two edges of the edge sum extended to $\mathrm{L}$ events to the left and right end sides. $\mathrm{L}$ is

${ }^{7}$ measured by the DIADOS, a diagnostic dosemeter produced by PTW-
Freiburg

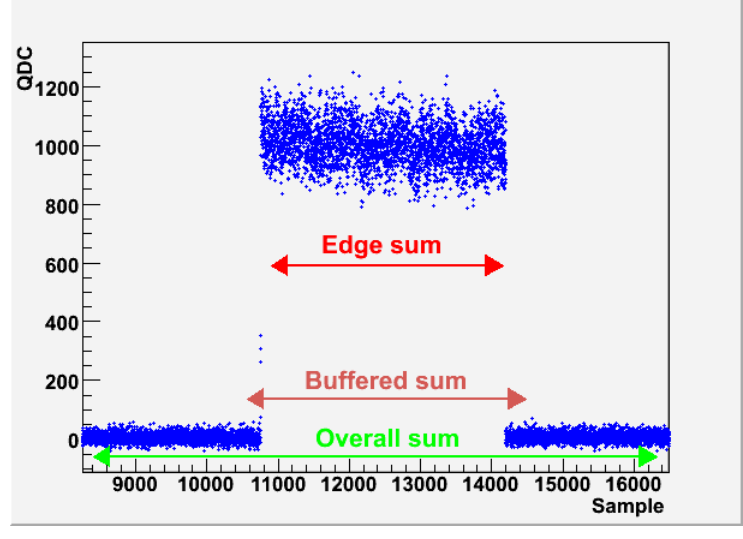

Fig. 2. The output signal, pedestal subtracted, for a typical data set is shown as a function of the sample number. The set of events considered for the definition of the overall, buffered and edge sums are indicated.

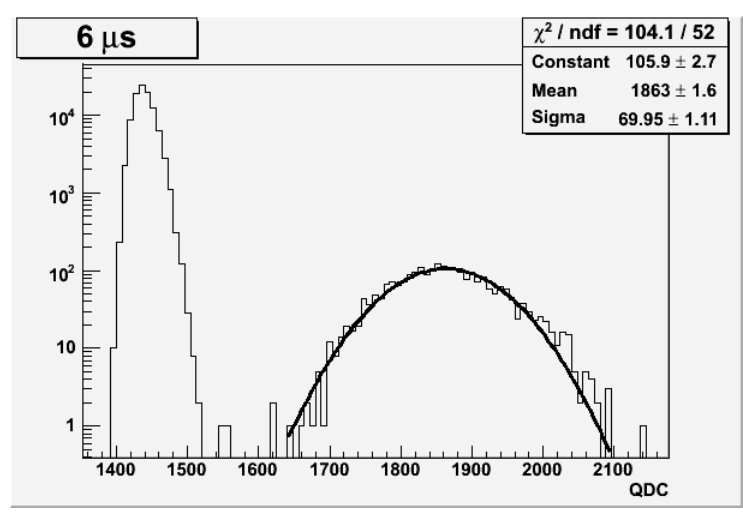

Fig. 3. Pedestal and Signal QDC distributions for a typical data set. The measurement refer to the fiber setup and the Hamamatsu sensor. The integration time for the reported data was $6000 \mathrm{~ns}$ for a dose rate of $2 \mathrm{mGy} / \mathrm{s}$. The gaussian fit is restricted to a range of values exceeding by 3.5 sigma the pedestal.

typically 20 . The buffer samples are added in order to get rid of any bias in the edge definition and their number should be kept as small as possible not to introduce further inaccuracy.

- the mean of a Gaussian fit performed on the distribution of beam-on events (see Fig. 3).

- the edge mean, calculated dividing the edge sum by the number of considered samples.

- the buffered mean, obtained dividing the buffered sum by the number of considered samples.

For each quantity, the offset and its spread were measured using beam-off samples.

The system response to increasing dose rates has been measured and compared to the values provided by the UNIDOS. The precision and the linearity have been evaluated from these sets of measurements using both the tile and the fiber set-up. As exemplary illustration, the linear correlation between the two independent measurements is shown in Fig. 4 for the tile 


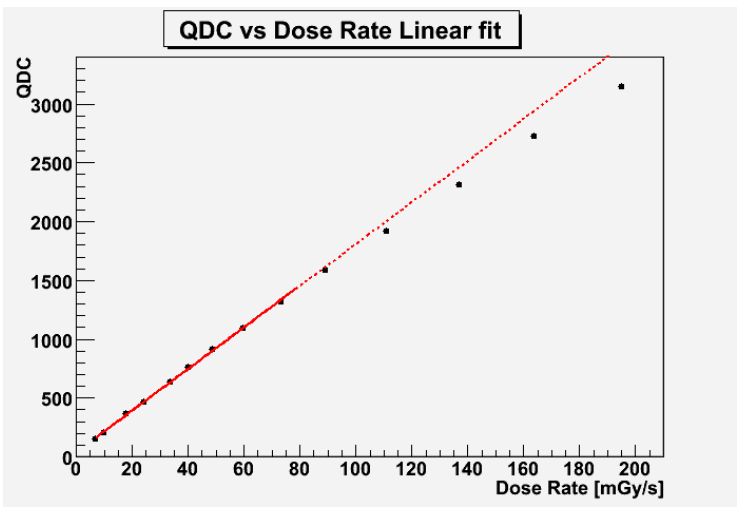

Fig. 4. Linearity of the sensor response (in terms of Most Probable Value) for increasing dose rates. Measurements refer to the tile coupled to the SensL sensor set up.

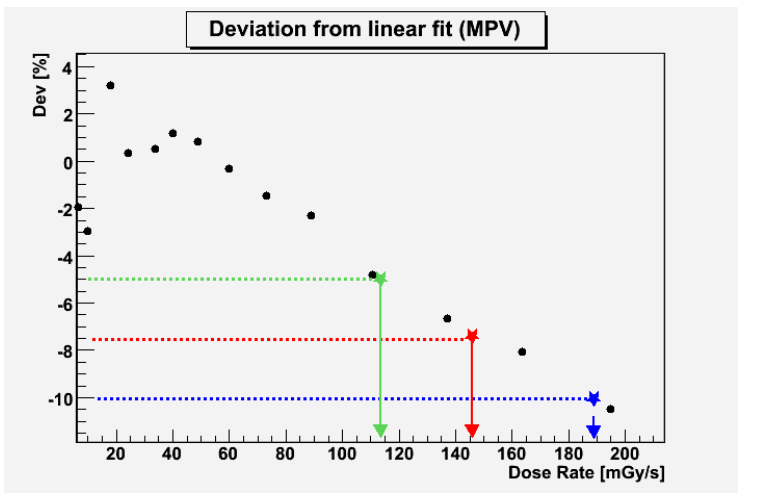

Fig. 5. Percentage deviation from the linear fit as a function of the dose rate. Measurements refer to the $1 \mathrm{~mm}$ tile coupled to the SensL sensor set up.

set-up. The precision is computed as the standard deviation of the measurements, corrected for the correlation factor, while the linearity was evaluated fitting the data up to $80 \mathrm{mGy} / \mathrm{s}$ and then plotting the trend of the deviations from the extrapolated linear fit as a function of the dose rate up to $200 \mathrm{mGy} / \mathrm{s}$, as shown in Fig. 5. The dose rates corresponding to 5\%, 7.5\% and $10 \%$ level have been computed and retained as indicators. The precision was also cross-checked by measuring the standard deviation of a set of 50 measurements recorded at the same machine settings, accounting for the machine instabilites by a direct independent measurement of the dose by the UNIDOS.

Moreover, from the dose rate linear fit it is possible to evaluate the sensitivity $(S)$ and the minimum detectable signal $(M D S)$, relying on the following equations :

$$
\begin{gathered}
S=N \cdot \frac{\sigma_{y}}{a} \\
M D S=N \cdot \frac{\sqrt{\sigma_{\text {pede }}^{2}+\sigma_{\text {signal }}^{2}}}{a}
\end{gathered}
$$

where

- $\mathrm{N}$ is a user defined integer, assumed here to be 1
- a is the slope of the linearity fit

- $\sigma_{\text {pede }}, \sigma_{\text {signal }}$ and $\sigma_{y}$ are respectively the standard deviation of the offset, of the lowest dose rate measurements and the spread of the measurements at the generic dose rate.

A summary of the system performances, for the three configurations, is reported in Tab II Tab III and Tab IV. The sensitivity was estimated at the mid value of the dose rate range.

\section{CONCLUSION AND OUTLOOK}

The RAPSODI project has successfully entered its last development phase. Results do confirm the potential for in-vivo, real-time dosimetry in mammography. In particular, it has been proven that the system is compliant to the requirements of the council directive 97/43 EURATOM (article 8, sub-clause 6). The final prototype engineering phase has already been started with the main goal to minimise the X-ray image artifacts. By the end of the project working prototypes for the forseen applications will be ready and completely qualified. Moreover, dedicated electronics boards and ASICs are beeing developed in order to replace the laboratory VME based DAQ system nowadays in use to digitise the SiPM produced signals.

\section{REFERENCES}

[1] A.Akindinov et al., "New results on MRS APDs", Nucl. Inst. and Meth.A 387 (1997) 231-234 


\begin{tabular}{lcccccc}
\hline \hline Observable & $\begin{array}{c}\text { Precision } \\
{[\%]}\end{array}$ & $\begin{array}{c}\text { Gain } \\
{[\mathrm{QDC} / \mathrm{mGy} / \mathrm{s}]}\end{array}$ & $\begin{array}{c}\text { Sensitivity } \\
{[\mathrm{mGy} / \mathrm{s}]}\end{array}$ & $\begin{array}{c}\text { MDS } \\
{[\mathrm{mGy} / \mathrm{s}]}\end{array}$ & $\begin{array}{c}\text { Lin. range } \\
\leq 5 \%[\mathrm{mGy} / \mathrm{s}]\end{array}$ & $\begin{array}{c}\text { Lin. range } \\
\leq 7.5 \%[\mathrm{mGy} / \mathrm{s}]\end{array}$ \\
\hline Overall sum & $3.10 \pm 0.04$ & $57.48 \pm 0.66$ & $2.76 \pm 0.05$ & $0.725 \pm 0.014$ & 170 & 200 \\
Edge sum & $2.33 \pm 0.03$ & $57.68 \pm 0.49$ & $2.06 \pm 0.03$ & $0.459 \pm 0.007$ & 170 & 200 \\
Buff. sum & $2.31 \pm 0.03$ & $57.69 \pm 0.49$ & $2.05 \pm 0.03$ & $0.458 \pm 0.007$ & 170 & 200 \\
Mean & $2.07 \pm 0.03$ & $0.01691 \pm 0.00001$ & $1.82 \pm 0.02$ & $0.216 \pm 0.002$ & 170 & 200 \\
Edge mean & $2.08 \pm 0.03$ & $0.01649 \pm 0.0001$ & $1.88 \pm 0.03$ & $0.236 \pm 0.002$ & 170 & 200 \\
Buff. mean & $2.12 \pm 0.03$ & $0.01636 \pm 0.0001$ & $1.92 \pm 0.03$ & $0.234 \pm 0.002$ & 170 & 200 \\
\hline \hline
\end{tabular}

TABLE II

CHARACTERISATION RESULTS FOR THE PROTOTYPE WITH SCINTILLATING FIBER COUPLED TO THE HAMAMATSU SENSOR.

\begin{tabular}{lccccc}
\hline \hline Observable & $\begin{array}{c}\text { Precision } \\
{[\%]}\end{array}$ & $\begin{array}{c}\text { Gain } \\
{[\mathrm{QDC} / \mathrm{mGy} / \mathrm{s}]}\end{array}$ & $\begin{array}{c}\text { Sensitivity } \\
{[\mathrm{mGy} / \mathrm{s}]}\end{array}$ & $\begin{array}{c}\text { MDS } \\
{[\mathrm{mGy} / \mathrm{s}]}\end{array}$ & $\begin{array}{c}\text { Lin. range } \\
\leq 5 \%[\mathrm{mGy} / \mathrm{s}]\end{array}$ \\
\hline Overall sum & $27.58 \pm 0.7$ & $13.57 \pm 2.11$ & $7.04 \pm 1.11$ & $7.044 \pm 1.11$ & 40 \\
Edge sum & $1.99 \pm 0.05$ & $14.30 \pm 0.14$ & $0.46 \pm 0.01$ & $0.460 \pm 0.01$ & 40 \\
Buff. sum & $1.95 \pm 0.05$ & $14.32 \pm 0.14$ & $0.45 \pm 0.01$ & $0.452 \pm 0.01$ & 40 \\
Mean & $1.95 \pm 0.05$ & $0.00500 \pm 0.00001$ & $0.45 \pm 0.01$ & $2.378 \pm 0.05$ & 40 \\
Edge mean & $1.81 \pm 0.05$ & $0.00499 \pm 0.00005$ & $0.42 \pm 0.01$ & $2.355 \pm 0.05$ & 40 \\
Buff. mean & $1.80 \pm 0.05$ & $0.00494 \pm 0.00005$ & $0.42 \pm 0.01$ & $2.370 \pm 0.05$ & 40 \\
\hline \hline
\end{tabular}

TABLE III

CHARACTERISATION RESUlTS FOR THE PROTOTYPE WITH SCINTILLATING FIBER COUPLED TO THE SENSL SENSOR.

\begin{tabular}{|c|c|c|c|c|c|c|c|}
\hline Observable & $\begin{array}{c}\text { Precision } \\
{[\%]}\end{array}$ & $\begin{array}{c}\text { Gain } \\
{[\mathrm{QDC} / \mathrm{mGy} / \mathrm{s}]}\end{array}$ & $\begin{array}{l}\text { Sensitivity } \\
{[\mathrm{mGy} / \mathrm{s}]}\end{array}$ & $\begin{array}{c}\text { MDS } \\
{[\mathrm{mGy} / \mathrm{s}]}\end{array}$ & $\begin{aligned} & \text { Lin. range } \\
\leq & 5 \%[\mathrm{mGy} / \mathrm{s}]\end{aligned}$ & $\begin{array}{c}\text { Lin. range } \\
\leq 7.5 \%[\mathrm{mGy} / \mathrm{s}]\end{array}$ & $\begin{aligned} & \text { Lin. range } \\
\leq & 10 \%[\mathrm{mGy} / \mathrm{s}]\end{aligned}$ \\
\hline Overall sum & $3.60 \pm 0.05$ & $111.32 \pm 1.43$ & $3.17 \pm 0.06$ & $0.864 \pm 0.018$ & 115 & 155 & 190 \\
\hline Edge sum & $3.08 \pm 0.05$ & $111.80 \pm 1.19$ & $2.63 \pm 0.05$ & $0.527 \pm 0.009$ & 115 & 165 & 195 \\
\hline Buff. sum & $3.04 \pm 0.05$ & $112.05 \pm 1.18$ & $2.60 \pm 0.05$ & $0.524 \pm 0.009$ & 150 & 160 & 195 \\
\hline Mean & $3.17 \pm 0.05$ & $0.03286 \pm 0.00001$ & $2.67 \pm 0.04$ & $0.723 \pm 0.007$ & 115 & 155 & 190 \\
\hline Edge mean & $2.99 \pm 0.04$ & $0.03192 \pm 0.0003$ & $2.60 \pm 0.05$ & $0.720 \pm 0.007$ & 115 & 165 & 195 \\
\hline Buff. mean & $2.95 \pm 0.04$ & $0.03173 \pm 0.0003$ & $2.56 \pm 0.05$ & $0.718 \pm 0.007$ & 115 & 165 & 195 \\
\hline
\end{tabular}

TABLE IV

CHARACTERISATION RESULTS FOR THE PROTOTYPE WITH SCINTILLATING TILE COUPLED TO THE SENSL SENSOR. 